\title{
Studying activity and toxicity of different TNF-alpha inhibitors in controlling patients with active ulcerative colitis
}

\author{
Bahir Abdul Razzaq Mshimesh* \\ Department of Pharmacology \& Toxicology, College of Pharmacy, Mustansiriyah University, Baghdad, Iraq.
}

\begin{tabular}{|c|c|}
\hline ARTICLE INFO & ABSTRACT \\
\hline $\begin{array}{l}\text { Received on: } 29 / 10 / 2018 \\
\text { Accepted on: } 15 / 12 / 2018 \\
\text { Available online: } 28 / 02 / 2019\end{array}$ & $\begin{array}{l}\text { Ulcerative colitis (UC) is a long-term disorder that associated with overactivation of immunoinflammatory system, } \\
\text { ending with ulcer in the large intestine. This study aimed to compare the activity and toxicity of different TNF-alpha } \\
\text { inhibitors in a sample of Iraqi patients having active ulcerative colitis. Fifty patients with refractory ulcerative colitis } \\
\text { were randomly distributed to either group I }(n=25) \text { : on adalimumab injection }(160 / 80 \mathrm{mg}) \text { or group II }(n=25) \text { : on }\end{array}$ \\
\hline $\begin{array}{l}\text { Key words: } \\
\text { Ulcerative colitis, clinical } \\
\text { remission, safety profile, } \\
\text { adalimumab, infliximab. }\end{array}$ & $\begin{array}{l}\text { infliximab injection }(5 \mathrm{mg} / \mathrm{kg}) \text { along the term of induction. Clinical remission was considered as the primary subjective } \\
\text { parameter, while clinical response, mucosal healing, and subscores of mild status were regarded as secondary subjective } \\
\text { parameters. Fractional Mayo score, inflammatory bowel disease questionnaire (IBDQ), and safety profile were also } \\
\text { evaluated. A } 24 \% \text { versus } 28 \% \text { patients were in clinical remission for those receiving adalimumab and infliximab, } \\
\text { respectively }(p>0.05) \text {, while } 48 \% \text { versus } 52 \% \text { patients were in clinical response for those receiving adalimumab and } \\
\text { infliximab, respectively }(p>0.05) \text {, and } 40 \% \text { of patients acquired mucosal healing for both adalimumab and infliximab } \\
\text { arms }(p>0.05) \text {. Scores of physician assessment and rectal bleeding was shown to be markedly higher in infliximab } \\
\text { patients }(p<0.05) \text {, while those of stool frequency was found to be higher in adalimumab patients }(p<0.05) \text {. No } \\
\text { significant difference was observed between two arms in the fractional Mayo score and IBDQ index }(p>0.05) \text {. } \\
\text { Both of the studied biological agents were well-tolerated. As conclusion, the two different TNF-alpha inhibitors were } \\
\text { comparable in their clinical remission and safety profile for subjects with active ulcerative colitis. }\end{array}$ \\
\hline
\end{tabular}

\section{INTRODUCTION}

Ulcerative colitis (UC) is an idiopathic, chronic inflammatory disease of the digestive organ, for the most part including the rectum, described by a persistent irritation and ulceration of the mucosa and submucosa. Displaying side effects incorporate rectal bleeding, looseness of the bowels, urgency, and bowel pain (Rubin et al., 2010). UC has a critical negative effect on quiet personal satisfaction and spots a considerable money related weight on medicinal services frameworks, with an estimated cost exceeding $\$ 3.4$ billion in the United State and $€ 5.4$ billion in Europe. Objectives of treatment incorporate the acceptance

\section{*Corresponding Author}

Bahir Abdul Razzaq Mshimesh, Department of Pharmacology \& Toxicology, College of Pharmacy, Mustansiriyah University, Baghdad,Iraq.E-mail:dr.bahirrazzaq@gmail.com, dr.bahirrazzq@uomustansiriyah.edu.iq and support of remission, enhanced personal satisfaction, and reduction of illness and treatment-related events (Cohen et al., 2010).

Standard treatments incorporate 5-aminosalicylic acid, steroids and oral immunosuppressants (azathioprine, 6-mercaptopurine, and cyclosporine). These drugs deficiently control UC to a considerable extent of patients and can prompt unfavorable occasions and adverse drug reactions (AEs). In this way, there is a requirement for new treatments past traditional treatment choices for some patients with ulcerative colitis (Cohen et al., 2010; Rubin et al., 2010). Tumor necrosis factor-alpha (TNF-alpha) is a normally happening proinflammatory cytokine that seems to assume a basic part in the pathogenic procedures of ulcerative colitis (Sartor, 1994). TNF-alpha production is expanded in mucosal macrophages detached from the lesion, and it shown in expanded levels in the blood circulation, mucosal tissue, and stools of those with ulcerative colitis (Braegger et al., 1992; Murch et al., 1991; 1993). 
Within the recent decade, a randomized controlled preliminary studies have demonstrated the counter treatment of TNF, infliximab (IFX, a chimeric IgG1 monoclonal antibody), and adalimumab (ADA, a fully humanized IgG1 monoclonal antibody) to be viable in controlling of ulcerative colitis (Reinisch et al., 2011; Rutgeerts et al., 2005), notwithstanding, in Crohn's illness and another immune system issue (Hinojosa et al., 2007; Teshima et al., 2009). These anti-TNF medicines work by promoting apoptosis of the TNF-alpha-communicating provocative cells, canceling the soluble TNF, and also exhaust the number of insusceptible cells through antibody-dependent cell-mediated and complement-dependent cytotoxicity. Anti-TNF treatments, infliximab or adalimumab, are for the most part held for the treatment of moderate-severe inflammatory bowel disorders (IBD) that have not reacted to corticosteroids andor immunosuppressive therapies, or when the patient encounters an unfavorable occasion or unfit to endure corticosteroids andor immunosuppressive drugs (Chowers and Allez, 2010).

The reason for the present trial was to analyze the viability and tolerability of adalimumab and infliximab for induction of remission in Iraqi patients with moderate-severe ulcerative colitis by a "real" clinical practice conditions.

\section{METHODS}

\section{Patient's selection}

This randomized double-blind comparative study was conducted at Baghdad Centers of GI and Liver Disorders between April and November 2016. The protocol was achieved under the supervision of specialist physicians and approved by the ethical committee of the College of Pharmacy/Mustansiryiah University. The procedures followed were in accordance with the ethical standards of the responsible committee on human experimentation and with the Helsinki Declaration. All patients gave oral and written consent.

Eligible patients were ambulatory subjects with a moderate-severe ulcerative colitis for no less than 3 months with a Mayo score of (7-12) items and endoscopy subscore of $\geq 2$, in spite of simultaneous treatment with steroids or potentially azathioprine or 6-mercaptopurine. The Mayo score composed of four things [rectal bleeding score (RBS), stool frequency score (SFS), physician global assessment (PGA), and mucosal appearance)] (Table 1) (Schroeder et al., 1987).

For the scoring of the rectal bleeding and stool recurrence index, the most noticeably bad score from the past 3 days before the examination visit was utilized. The analysis of ulcerative colitis was affirmed by biopsy got at the screening colonoscopy or adaptable sigmoidoscopy. Patients simultaneously treated with oral corticosteroids were to get a steady measurement (prednisone $\geq 20 \mathrm{mg} /$ day for no less than 2 weeks or $<20 \mathrm{mg}$ /day for no less than 40 days) before baseline. Patients treated with immunomodulators were to get no less than a continuous 3 -month course of azathioprine $(1.5 \mathrm{mg} / \mathrm{kg} /$ day or maximum endured dose) or 6-mercaptopurine $(1 \mathrm{mg} / \mathrm{kg} /$ day or maximum endured dose) before baseline (with stable dose for no less than a month). Simultaneous treatment was not required for patients who neglected to react to or couldn't endure past corticosteroid or immunomodulator treatment. Patients were permitted stable doses of 5-aminosalicylates as simultaneous treatment; however, 5-aminosalicylate utilizes was not a mandatory requirement for this study. Past utilization of ant-TNF drugs was not allowed. Female gender patients were postmenopausal, sterilized with the operation, or utilizing anti-conception medication.

Patients not included if they had: a history of colectomy with ileorectostomy for ulcerative colitis or arranged inside medical procedure; past treatment with anti-TNF therapy; receipt of IV corticosteroids in 2 weeks of screening; immunosuppressants in 4 weeks of baseline; bowel purge or suppository in 2 weeks of the screening endoscopy and within the screening time; any diagnostic substance during 4 weeks; pregnant and lactant ladies; fulminant colitis or megacolon; rectum disorders (e.g., ulcerative proctitis) or history of Crohn's disease; current parenteral nourishment; Clostridium difficile positive stool; history of IV antimicrobial treatment in 4 weeks or oral antimicrobial treatment in 2 weeks (e.g., ciprofloxacin and metronidazole); history of histoplasmosis; hepatitis B disease; AIDS or untreated TB affirmed by radiology or skin test; live vaccine in 3 months before baseline; history of cancer; inadequately controlled conditions (e.g., diabetes) and known allergy to adalimumab or infliximab formula.

\section{Study design}

Patients randomly assigned in 1:1 proportion to get either adalimumab or infliximab. Adalimumab-treated patients got SC dosage at 0 weeks, 2 weeks, and a month of 160,80 , and $40 \mathrm{mg}$, respectively, then $40 \mathrm{mg}$ each other week. Infliximabtreated patients got IV dosage of $5 \mathrm{mg} / \mathrm{kg}$ at 0 weeks, 2 weeks and 6 weeks, then a support dosage of $5 \mathrm{mgkg}$ each other week. Incomplete response was characterized by (1) fractional Mayo score $\geq$ baseline score on two successive visits no less than 2 weeks separated (for patients with a fractional Mayo score of 4-7 at baseline); (2) fractional Mayo score $\geq 7$ on two successive visits no less than 2 weeks separated (for patients with a fractional Mayo score of 8-9 at baseline). Other medications continued with the exception of steroids, which could be decreased after week 8 in patients who had an attractive clinical response.

\section{Efficacy evaluation}

\section{Primary and secondary variables}

These variables involve the fraction of remission patients in each group per Mayo score at week 8 (remission characterized as Mayo score $\leq 2$ with no individual subscore $>1$ ). Secondary

Table 1. UC disease activity index (Schroeder et al., 1987).

\begin{tabular}{ll}
\hline SFS & Mucosal appearance \\
\hline Normal $=0$ & Normal $=0$ \\
$1-2$ stools $/$ day $>$ normal $=1$ & Mild friability $=1$ \\
$3-4$ stools $/$ day $>$ normal $=2$ & Moderate friability $=2$ \\
$>4$ stools $/$ day $>$ normal $=3$ & Exudation, spontaneous bleeding $=3$ \\
RBS & PGA \\
None $=0$ & Normal $=0$ \\
Streaks of blood $=1$ & Mild $=1$ \\
Obvious blood $=2$ & Moderate $=2$ \\
Mostly blood $=3$ & Severe $=3$ \\
\hline
\end{tabular}

Each item ranged from $0-3$, so the total index was between $0-12$. $0-2$ : remission/3-6: mild/7-10: moderate/>10: severe. 
variables were evaluated at week 8 in each group and represent: fraction of patients with clinical response per Mayo score (response: diminish in Mayo Score $\geq 3$ points and $\geq 30 \%$ from baseline in addition to a lessening in the RBS $\geq 1$ or absolute RBS of 0 or 1 ); fraction of patients with mucosal healing (endoscopy subscore of 0 or 1); fraction of patients with subscores characteristic of mild illness (RBS $\leq 1, \mathrm{SFS} \leq 1$, and PGA subscore $\leq 1$ ). Fractional Mayo score represents a Mayo score without endoscopy, and the fraction of patients in remission per fractional Mayo score (characterized as partial Mayo score $\leq 2$; with no subscore $>1$ ) was resolved in the two groups at weeks $0,2,4,6$, and 8 . Wellbeing related personal satisfaction, as estimated by the inflammatory bowel disease questionnaire (IBDQ), was additionally decided at weeks 0,2 , 4,6 , and 8 . The questionnaire response was characterized as an expansion from a baseline of $\geq 16$ points (Irvine et al., 1994).

\section{Additional analysis}

The fraction of patients remission per Mayo score at week 8 was surveyed after subgrouping by baseline Mayo scores (7-9 vs. 10-12), extent of disease, high sensitivity C-responsive protein level (hs-CRP $<10 \mathrm{mg} / 1 \mathrm{vs}$. hs-CRP $\geq 10 \mathrm{mg} / \mathrm{l}$ ], patients weight ( $<70 \mathrm{~kg} v s . \geq 70 \mathrm{~kg}$ ), and by accompanying drugs utilized (corticosteroid, immunomodulators, and aminosalicylates).

\section{Safety evaluation}

For each visit during this study, patients experienced a clinical examination, vital signs, and recording past or accompanying drugs. Unfavorable occasions and adverse events (AEs) were recorded and important lab investigations were achieved.

\section{Statistical analysis}

Demographics and baseline characteristics were summarized using descriptive statistics. Continuous variables were expressed as mean \pm standard error of the mean (SD), while categorical variables were expressed as frequencies and percentages. Continuous variables were compared using analysis of variance, discrete variables using Chi-square. Efficacy variables and partial Mayo scores were assessed in both patient groups. Results for the adalimumab and infliximab groups were compared using the Chi-square test for the evaluated endpoints; remission rates were analyzed using the same method. The subgroup analyses and incidence of adverse effects in the adalimumab group was compared with that of infliximab group using Fisher's exact test. All of the analyses were performed using the SPSS program and $p$-value $<0.05$ was considered statistically significant.

\section{RESULTS}

\section{Patients flow}

Of the 64 patients enrolled in this study (33 on adalimumab and 31 on infliximab), 50 completed 8 weeks ( 25 on adalimumab and 25 on infliximab). Reasons for discontinuation were summarized in Table 2.

\section{Baseline characteristics}

The baseline demographic and clinical features of patients were practically identical over the two treatment groups
(Table 3). Study members were mainly male, with a mean period of moderate-severe ulcerative colitis of around 6 years and Mayo score around 9 at baseline. Patients with infliximab had a numerically longer period of illness, a larger fraction of pancolitis, more mean Mayo score, and higher mean hs-CRP levels during baseline, however, the distinctions were not measurable $(p>0.05)$.

At baseline, total patients in both groups were taking medications with $50 \%$ on steroids, $16 \%$ receiving immunomodulators, $14 \%$ on steroids plus immunomodulators, and $82 \%$ receiving aminosalicylates. The higher users of steroid therapy at baseline were in the infliximab arm, while the percentage of patients used immunomodulators was the same for both arms (Table 3).

\section{Efficacy}

\section{Primary and secondary endpoints}

The patient's fraction with clinical remission in the adalimumab and infliximab groups was an approach $(p>0.05)$. At week $8,24 \%$ of patients who received adalimumab versus $28 \%$ of patients who received infliximab, were in clinical remission (Table 4, Fig. 1). Clinical response was reported at week 8 in $48 \%$ for those taking adalimumab and $52 \%$ for those on infliximab $(p>$ 0.05 ) (Fig. 1). Considering mucosal healing, it was seen at week 8 in $40 \%$ of patients receiving either adalimumab or infliximab $(p>$ $0.05)$ (Fig. 1). For the subscores indicative of mild disease $(\leq 1)$, the patients $\%$ of RBS and PGA was significantly higher within infliximab group $(p<0.05)$, while the patients $\%$ of SFS was significantly higher within adalimumab group $(p<0.05)$ (Table 4$)$.

The extent of patients accomplishing clinical remission in view of the fractional Mayo score for the two groups appears in Figure 2. Partial Mayo scores were utilized to measure remission induction with time. The extent of patients in light of this remission (fractional Mayo score $\leq 2$; with no subscore $>1$ ) expanded with time in the two arms, with no significant gap between the two biologic agents from week 2 through week 8 , reaching a maximum at week $8(p>0.05)$. Same results were observed for IBDQ response index ( $p>0.05)$ (Fig. 3). No patient achieved steroidfree remission for both groups during the study period; also, no patient underwent colectomy for both agents.

\section{Additional analysis}

At baseline, those with Mayo score $\geq 10$ had less extent of remission versus those having Mayo score $<10$ in both groups, although it was more pronounced in the infliximab group (Table 5). Effects of treatment were approximated in those with

Table 2. Reasons for discontinuation of treatment.

\begin{tabular}{lcc}
\hline Reason of withdrawal & ADA group $(\boldsymbol{n}=\mathbf{3 3})$ & IFX group $(\boldsymbol{n}=\mathbf{3 1})$ \\
\hline Consent withdraw & $2(6)$ & $1(3)$ \\
Lack of efficacy & $1(3)$ & 0 \\
Adverse events & $2(6)$ & $2(6)$ \\
Protocol violation & $2(6)$ & $1(3)$ \\
Lost to follow up & $1(3)$ & $2(6)$ \\
Death & 0 & 0 \\
\hline
\end{tabular}

$\mathrm{ADA}=$ adalimumab, IFX $=$ infliximab. Data expressed by $n(\%), n=$ no. of patients. 
or without extensive colitis, while higher hs-CRP levels $(\geq 10$ $\mathrm{mg} / \mathrm{l}$ or $95.238 \mathrm{nmol} / \mathrm{l})$ and higher patient's weight $(\geq 70 \mathrm{~kg})$ at baseline were associated with reduced remission rates. However, treatment effects were more pronounced in patients treated with corticosteroids plus immunosuppressants, and in those who received aminosalicylates at baseline.

\section{Safety analysis}

Adalimumab and infliximab were generally endurable and the safety profile was matched. A comparable fraction of patients in each arm showed drug therapy-adverse events (Table 6). Most of these adverse events were not serious, mild or moderate in severity, and were considered "not related" or "probably not related" to the study drugs.

No considerable difference was found between both arms for most of the emergent adverse events $(p>0.05)$. However, the incidence of severe and serious adverse events tended to be numerically higher in one biologic agent over the other.

The extent of patients who ended the research on account of undesired occasions was low and comparative over both groups. One patient in adalimumab arm showed exacerbating or flare of ulcerative colitis. All infusion site responses were gentle and most were overseen without considered interference or drug stop. The occurrence of infusion site pain was essentially higher in the infliximab arm $(p<0.05)$.

Serious infections were reported in one patient of the adalimumab group (wound infection) and one patient in the infliximab group (pneumonia), while just one patient in the infliximab group experienced an opportunistic infection (esophageal candidiasis). One patient (70 years) was accounted with TB for a not long time after the last dosage of adalimumab and on prednisone $20 \mathrm{mg} /$ day; this patient had given a negative skin test and chest X-ray at baseline. The patient was referred for the treatment

A significant proportion of infliximab-treated patients reported blood-related adverse reactions, in contrast to adalimumab-treated patients $(p<0.05)$. The last unfavorable occasions (especially leukopenia) were accounted for in the infliximab-treated patients who were taking immunosuppressants at the baseline level. All occasions were settled before finishing the trial. There were no mortality cases, hepatic disorders, lymphoma, melanoma or other malignancies, cardiac problems, neuronal, or lupus-like disorders announced in each group. Investigations of lab tests and essential signs did not show any extra safety problems.

Table 3. Baseline clinical and demographic characteristics of studied groups.

\begin{tabular}{|c|c|c|c|}
\hline Characteristics & ADA group $(n=25)$ & IFX group $(n=25)$ & Total $(n=50)$ \\
\hline Age, $y, \mathrm{M} \pm \mathrm{SD}$ & $42.3 \pm 12.22$ & $40.6 \pm 14.47$ & $41.4 \pm 13.86$ \\
\hline Male, $n(\%)$ & $18(72)$ & $16(64)$ & $34(68)$ \\
\hline Weight, $\mathrm{kg}, \mathrm{M} \pm \mathrm{SD}$ & $76.1 \pm 16.31$ & $74.3 \pm 18.71$ & $75.2 \pm 17.52$ \\
\hline Smokers, $n(\%)$ & $7(28)$ & $9(36)$ & $16(32)$ \\
\hline Disease duration, $y, \mathrm{M} \pm \mathrm{SD}$ & $6.1 \pm 5.37$ & $6.5 \pm 5.09$ & $6.3 \pm 5.23$ \\
\hline Previous relapse, $\mathrm{M} \pm \mathrm{SD}$ & $3.2 \pm 1.06$ & $3.3 \pm 1.02$ & $3.25 \pm 1.04$ \\
\hline \multicolumn{4}{|l|}{ Site of UC, $n(\%)$} \\
\hline Pancolitis & $14(56)$ & $16(64)$ & $30(60)$ \\
\hline Descending colon & $10(40)$ & $8(32)$ & $18(36)$ \\
\hline Other & $1(4)$ & $3(12)$ & $4(8)$ \\
\hline Extra-intestinal findings & $6(24)$ & $8(32)$ & $14(28)$ \\
\hline Mayo score, $\mathrm{M} \pm \mathrm{SD}$ & $8.8 \pm 1.50$ & $9.0 \pm 2.0$ & $8.90 \pm 1.75$ \\
\hline Endoscopy & $2.4 \pm 0.50$ & $2.5 \pm 0.50$ & $2.45 \pm 0.50$ \\
\hline RBS & $1.7 \pm 0.85$ & $1.6 \pm 0.95$ & $1.65 \pm 0.90$ \\
\hline PGA & $2.2 \pm 0.75$ & $2.3 \pm 0.25$ & $2.25 \pm 0.50$ \\
\hline SFS & $2.5 \pm 0.70$ & $2.6 \pm 0.60$ & $2.55 \pm 0.65$ \\
\hline Partial Mayo score & $6.4 \pm 1.35$ & $6.5 \pm 1.55$ & $6.45 \pm 1.45$ \\
\hline IBDQ index, $\mathrm{M} \pm \mathrm{SD}$ & $150.2 \pm 26.9$ & $146.9 \pm 28.7$ & $148.0 \pm 27.8$ \\
\hline \multicolumn{4}{|l|}{ hs-CRP, $\mathrm{M} \pm \mathrm{SD}$} \\
\hline $\mathrm{mg} / \mathrm{l}$ & $13.7 \pm 38.78$ & $14.3 \pm 30.07$ & $14.0 \pm 34.48$ \\
\hline $\mathrm{nmol} / 1$ & $130.47 \pm 46.83$ & $136.19 \pm 45.05$ & $133.33 \pm 46.32$ \\
\hline \multicolumn{4}{|l|}{ Co-medication, $n(\%)$} \\
\hline Steroids & $12(48)$ & $13(52)$ & $25(50)$ \\
\hline IMM (Aza/6-MP) & $4(16)$ & $4(16)$ & $8(16)$ \\
\hline Steroids + IMM (Aza/6-MP) & $4(16)$ & $3(12)$ & $7(14)$ \\
\hline No steroids, no IMM & $5(20)$ & $5(20)$ & $10(20)$ \\
\hline Aminosalicylates (5-ASA) & $21(84)$ & $20(80)$ & $41(82)$ \\
\hline
\end{tabular}

$\mathrm{ADA}=$ adalimumab, IFX = infliximab, RBS $=$ rectal bleeding subscore, $\mathrm{PGA}=$ physician global assessment, SFS $=$ stool frequency subscore, IBDQ = inflammatory bowel disease questionnaire, hs-CRP $=$ high sensitivity C-reactive protein, $\mathrm{IMM}=$ immunomodulators, Aza $=$ azathioprine, 6 -MP $=6$-mercaptopurine. Data expressed by mean $\pm \mathrm{SD}$, or $n(\%)$, where $n=$ no. of patients. No significant differences $(p>0.05)$. 


\section{DISCUSSION}

This study has a similar inclusion and exclusion criteria to the previous European trials of infliximab and adalimumab (Rutgeerts et al., 2005; Sandborn et al., 2012). At the present, just restricted information is accessible on the "reality" of clinical practice results of anti-TNF therapy for ulcerative colitis. A small number of trials have archived open-label design with adalimumab and infliximab (Afif et al., 2009; Oussalah et al., 2008; Peyrin et al., 2007). Two huge randomized-controlled studies with infliximab in the induction and maintenance of ulcerative colitis

Table 4. Results of primary and secondary variables after 8 weeks of treatment.

\begin{tabular}{lcc}
\hline Variables & ADA group $(\boldsymbol{n}=\mathbf{2 5})$ & IFX group $(\boldsymbol{n}=\mathbf{2 5})$ \\
\hline Clinical remission & $6(24)$ & $7(28)$ \\
Clinical response & $12(48)$ & $13(52)$ \\
Mucosal healing & $10(40)$ & $10(40)$ \\
RBS $\leq 1$ & $16(64)$ & $20(80)^{*}$ \\
PGA $\leq 1$ & $12(48)$ & $15(60)^{*}$ \\
SFS $\leq 1$ & $13(52)^{*}$ & $9(36)$ \\
\hline
\end{tabular}

$\mathrm{ADA}=$ adalimumab, $\mathrm{IFX}=$ infliximab, $\mathrm{RBS}=$ rectal bleeding subscore, $\mathrm{PGA}=$ physician global assessment, SFS $=$ stool frequency subscore. Data expressed by $n(\%)$, where $n=$ no. of patients. *Significant difference $(p<0.05)$ had been reported (ACT1 and ACT2) (Rutgeerts et al., 2005) and two vast randomized-controlled studies with adalimumab in the induction and maintenance of ulcerative colitis had been accomplished (ULTRA1 and ULTRA2) (Reinisch et al., 2011; Sandborn et al., 2012).

In this study, treatment with adalimumab and infliximab demonstrated a substantial benefit in the clinical remission extent at week 8 (24\% vs. $28 \%$, respectively) among patients who were previously or currently not responded to steroids and/ or immunosuppressants. Substantial benefits were also seen for clinical response, partial Mayo score components, in addition to IBDQ index, compared with baseline data.

Two clinical reports depicting adalimumab therapy for ulcerative colitis have been documented: (1) which had a 13 cohort patients, demonstrated the likelihood of an adalimumab induce response being kept up as $84.6 \%$ at 3 months and $60.6 \%$ at a half year, (2) which revealed that $67 \%$ of a nine cohort patients had a considerable change in their disease status (Hudis et al., 2009; Oussalah et al., 2008). Essentially, the clinical practice utilization of infliximab for ulcerative colitis has been accounted for in three trials. In the initial, $67 \%$ of patients had an underlying clinical response, and $68 \%$ of these underlying responders encountered a maintained improvement (Ferrante et al., 2008), second trial

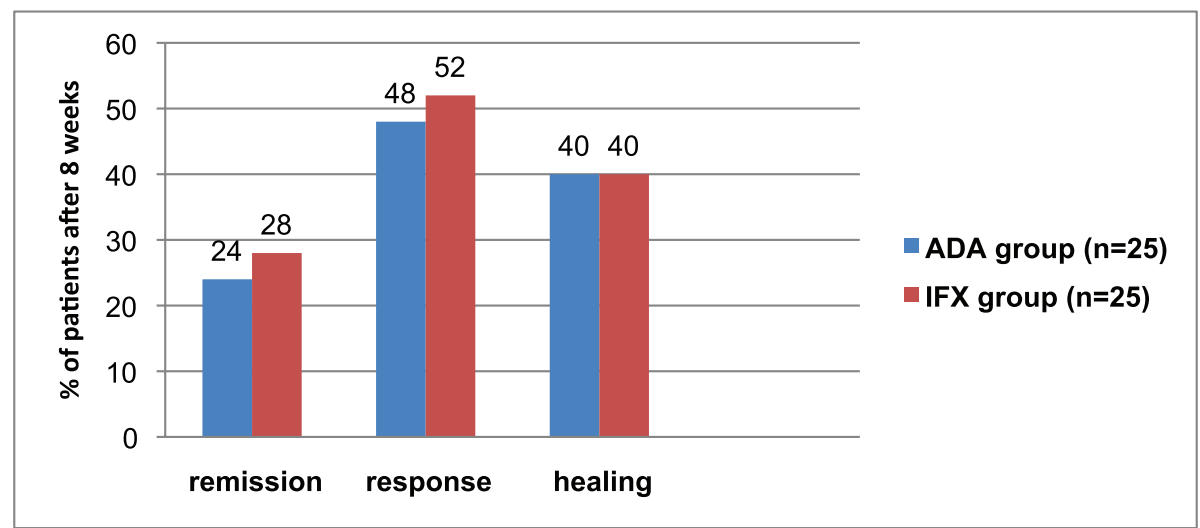

Figure 1. Fraction of patients with clinical remission, clinical response, and mucosal healing after 8 weeks of treatment with ADA or IFX agents. $n=$ no. of patients. $p>0.05$ means no significant difference.

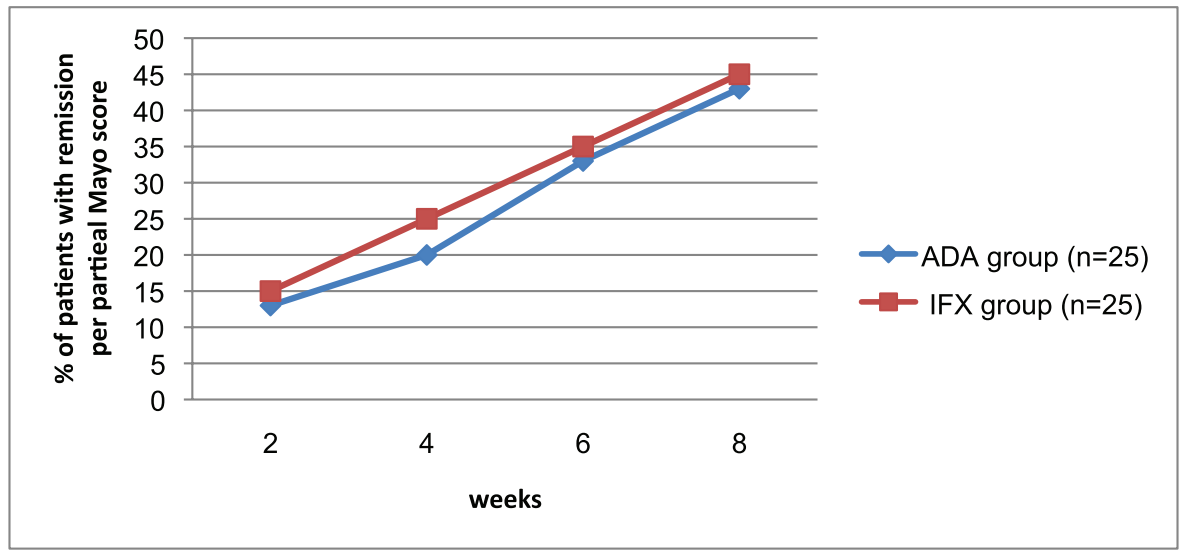

Figure 2. Proportion of patients achieving remission per partial Mayo score during 8 weeks of treatment with ADA or IFX agent. $n=$ no. of patients. $p>0.05$ means no significant difference. 


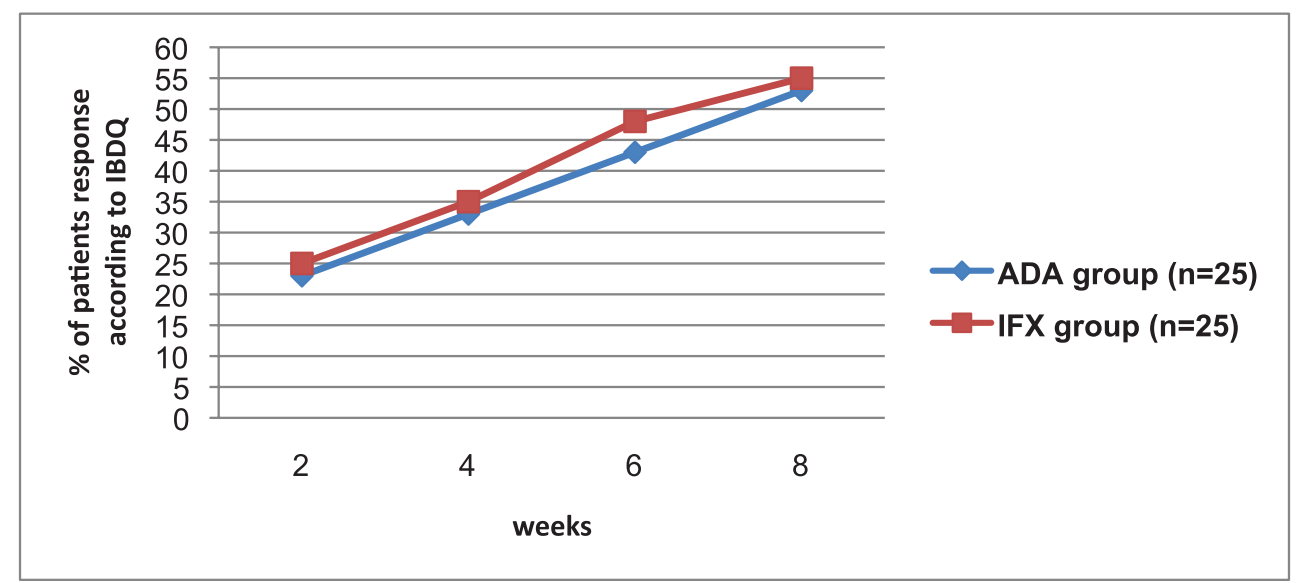

Figure 3. Proportion of patients achieving IBDQ response during 8 weeks of treatment with ADA or IFX agent. $n=$ no. of patients. $p>0.05$ means no significant difference.

demonstrated that the improvement rates were just $57 \%$ at week 8 (Senent et al., 2009), and the third one announced that clinical rate of response was $56 \%$ (Jürgens et al., 2009). The induction response rate in this "real-life" clinical practice study within 8 weeks was lower for infliximab (52\%) and adalimumab (48\%) than those revealed in the randomized placebo-controlled study for infliximab $(\mathrm{ACT} 1=69.4 \%$ and ACT2 $=64.5 \%$ ) (Rutgeerts et al., 2005) and adalimumab (ULTRA $1=54.6 \%$ and ULTRA $2=$ 50.4\%) (Reinisch et al., 2011; Sandborn et al., 2012).

Our outcomes consistent with the results of ULTRA1, an 8 -week induction study with adalimumab in those with ulcerative colitis which showed adalimumab 160/80 mg to be efficient for initiating clinical improvement. In that review, a measurable gap between adalimumab and placebo were accomplished for just two of the secondary variables at week 8, RBS and PGA (Reinisch et al., 2011). Interestingly, in ULTRA2, more prominent extent of adalimumab-treated patients accomplished all secondary variables at week 8 (Sandborn et al., 2012). The inconsistency between the two studies may because of the considerable response extent to placebo therapy that was seen with ULTRA1; while the response extent to placebo shown in ULTRA2 are for the most part like those revealed in the two substantial placebo-controlled studies of infliximab (ACT1 and ACT2) (Rutgeerts et al., 2005). Probably, a large number of clinic visits (3-5 visits/2 months) in these placebo-controlled studies may contribute to those outcomes through psychological considerations (Finniss et al., 2010).

As opposed to the infliximab reports, which showed that for remission induction of UC, $10 \mathrm{mg} / \mathrm{kg}$ of infliximab do not give more response than $5 \mathrm{mg} / \mathrm{kg}$ (Rutgeerts et al., 2005; Targan et al., 1997), dosing larger than $160 / 80 \mathrm{mg}$ of adalimumab did not tried. Dosing in the adalimumab induction studies for those with UC depended on the dosages of adalimumab known to be tolerable and efficient in Crohn's disorder (Colombel et al., 2007; Hanauer et al., 2006; Sandborn et al., 2007).

In the placebo groups within ACT1 and ACT2 trials, the clinical remission rates at week 8 were just $14.9 \%$ and $5.7 \%$, respectively, while for infliximab group $(5 \mathrm{mg} / \mathrm{kg})$ it was $38.8 \%$
Table 5. Results of the additional baseline subgroup analysis.

\begin{tabular}{|c|c|c|}
\hline Subgroups & ADA group $(n=25)$ & IFX group $(n=25)$ \\
\hline Mayo $<10, N$ & 16 & 17 \\
\hline Remission, $n(\%)$ & $5(31.3)$ & $7(41.2)$ \\
\hline Mayo $\geq 10, N$ & 9 & 8 \\
\hline Remission, $n(\%)$ & $1(11.1)^{*}$ & $2(25)^{*}$ \\
\hline Extensive colitis, $N$ & 13 & 12 \\
\hline Remission, $n(\%)$ & $3(23)$ & $4(33.3)$ \\
\hline No extensive colitis, $N$ & 12 & 13 \\
\hline Remission, $n(\%)$ & $4(33.3)$ & $3(23)$ \\
\hline hs-CRP $<10 \mathrm{mg} / 1$ or $95.238 \mathrm{nmol} / 1, N$ & 20 & 19 \\
\hline Remission, $n(\%)$ & $7(35)$ & $6(31.5)$ \\
\hline hs-CRP $\geq 10 \mathrm{mg} / 1$ or $95.238 \mathrm{nmol} / 1, N$ & 5 & 6 \\
\hline Remission, $n(\%)$ & $1(20)^{*}$ & $1(16.7)^{*}$ \\
\hline Weight $<70.0 \mathrm{~kg}, N$ & 15 & 16 \\
\hline Remission, $n(\%)$ & $6(40)$ & $6(37.5)$ \\
\hline Weight $\geq 70.0 \mathrm{~kg}, N$ & 10 & 9 \\
\hline Remission, $n(\%)$ & $2(20)^{*}$ & $1(11.1)^{*}$ \\
\hline Steroid (without IMM), $N$ & 12 & 13 \\
\hline Remission, $n(\%)$ & $3(25)$ & $4(30.7)$ \\
\hline IMM (without steroid), $N$ & 4 & 4 \\
\hline Remission, $n(\%)$ & $2(50)$ & $2(50)$ \\
\hline Steroid + IMM, $N$ & 4 & 3 \\
\hline Remission, $n(\%)$ & $3(75)$ & $2(66.7)$ \\
\hline No steroid + no IMM, $N$ & 5 & 5 \\
\hline Remission, $n(\%)$ & 0 & 0 \\
\hline Aminosalicylates, $N$ & 21 & 20 \\
\hline Remission, $n$ (\%) & $5(24)$ & $6(30)$ \\
\hline No aminosalicylates, $N$ & 4 & 5 \\
\hline Remission, $n(\%)$ & 0 & 0 \\
\hline
\end{tabular}

$\mathrm{ADA}=$ adalimumab, $\mathrm{IFX}=$ infliximab, hs-CRP $=$ high sensitivity C-reactive protein, $\mathrm{IMM}=$ immunomodulators. Data expressed by $n(\%)$, where $n=$ no. of patients. No significant difference between study groups $(p>0.05)$.*mean the significant difference between subgroups within the same group $(p<0.05)$. 
Table 6. Overview of the emergent adverse events for both groups.

\begin{tabular}{lcc}
\hline Adverse events & ADA group $(\boldsymbol{n}=\mathbf{2 5})$ & IFX group $(\boldsymbol{n}=\mathbf{2 5})$ \\
\hline Any & $20(80)$ & $21(84)$ \\
Possibly drug-related & $8(32)$ & $8(32)$ \\
Severe & $4(16)$ & $5(20)$ \\
Serious & $4(16)$ & $3(12)$ \\
Cause early withdrawal & $2(8)$ & $2(8)$ \\
Worsening/ flare of UC & $1(4)$ & 0 \\
hypersensitivity & $1(4)$ & $1(4)$ \\
Local infusion reaction & $2(8)$ & $5(20)^{*}$ \\
Serious infections & $1(4)$ & $1(4)$ \\
Opportunistic infection & 0 & $1(4)$ \\
Tuberculosis & $1(4)$ & 0 \\
Hematologic & $2(8)$ & $4(16)^{*}$ \\
Hepatic & 0 & 0 \\
Cardiac & 0 & 0 \\
Neuronal & 0 & 0 \\
Lupus-like & 0 & 0 \\
Malignant & 0 & 0 \\
Lymphomas & 0 & 0 \\
Fatal & 0 & 0 \\
\hline ADA & & 0
\end{tabular}

$\mathrm{ADA}=$ adalimumab, IFX $=$ infliximab. Data expressed by $n(\%)$, where $n=$ no. of patients. *Significant difference $(p<0.05)$.

and $33.9 \%$, respectively (Rutgeerts et al., 2005). In the present trial, the rate of remission to infliximab was just $28 \%$. Meanwhile, in the placebo groups within ACT1 and ACT2 trials, the clinical response rates at week 8 was just $37.2 \%$ and $29.3 \%$, respectively, while for infliximab group ( $5 \mathrm{mg} / \mathrm{kg}$ ) it was $69.4 \%$ and $64.5 \%$, respectively (Rutgeerts et al., 2005). In the present trial, the rate of response to infliximab was just 52\%. Likewise, patient's fraction who achieving mucosal healing in the placebo arm at week 8 was $33.9 \%$ in ACT1 and 30.9\% in ACT2, while for infliximab arm (5 $\mathrm{mg} / \mathrm{kg}$ ) it was $62.0 \%$ and $60.3 \%$, respectively (Rutgeerts et al., 2005). In the present trial, the rate of mucosal healing to infliximab was just $40 \%$.

In spite of the fact that the fundamental point of treatment for patients with ulcerative colitis has generally been relieving of symptoms, there is expanding confirmation to propose that reducing endoscopic lesion and accomplishing mucosal healing could be important for improving outcomes (Lichtenstein and Rutgeerts, 2010). In fact, healing of mucosa has been appeared to correspond with better remission, fewer complications and better satisfaction for patients (Colombel et al., 2011; Frøslie et al., 2007; Rutgeerts et al., 2007). The infliximab arm displayed essentially more extent of mucosal healing than placebo arm at weeks 8 and 30 in both ACT1 and ACT2 trials (Rutgeerts et al., 2005). Also, regarding the adalimumab population within ULTRA2 trial, the extent of healing for mucosa was higher than those in the placebo, both at week 8 and 52 (Sandborn et al., 2012).

Several trials have recommended that change in the extent of mucosal healing is related with a better outcome, enhanced personal satisfaction, and decreased the probability of colectomy (Colombel et al., 2011; Langholz, 2010; Lichtenstein and Rutgeerts, 2010). Utilizing information from ACT1 and ACT2, an ensuing report showed that patients treated with infliximab had an essentially bring down the rate of colectomy and hospital admission by week 54 than those on placebo (Sandborn et al., 2009). Utilizing information from ULTRA2, resulted outcomes have demonstrated that adalimumab treatment is related with diminished hospital admission and more prominent wellbeing and related personal satisfaction measures than placebo treatment, within 52 weeks (Feagan et al., 2014; Sandborn et al., 2011). In our study, no patient underwent colectomy or hospitalization throughout the follow-up period for both groups.

Later observational investigation for correlation of adalimumab versus infliximab-treated patients demonstrated good enhancement from baseline to follow up in response measures; consequences of these measures were comparative between adalimumab and infliximab participants. Variables like rectal bleeding, remission period, stool frequency, and PGA score showed no detectable differences between both agents (William et al., 2016). Authors concluded that Adalimumab and infliximab were similarly effective in the treatment of moderate to severe ulcerative colitis in the real-world clinical setting and this was consistent with the findings of the present study. However, not all secondary variables in this research were comparable between adalimumab and infliximab group, a meaningful difference was reported for RBS, PGA, and SFS.

In the present study and previous adalimumab trials, Mayo score was measured in view of the worst score from the most recent 3 days for rectal bleeding and stool frequency (Reinisch et al., 2011; Sandborn et al., 2012). Conversely, in the infliximab and other UC studies, the mean score for rectal bleeding and stool frequency from the most recent 3 days was utilized to measure Mayo score (Rutgeerts et al., 2005). What's more, subjects in the current trial were anti-TNF-naïve, though $40 \%$ of those in the adalimumab ULTRA2 were already presented with anti-TNF medications (Sandborn et al., 2012). The variations in the study plan and design may restrict comparisons.

Partial Mayo score information in this research show that the steady state level of response for adalimumab and infliximab had not yet been achieved at the end of week 8, proposing a requirement for a longer period to give the greatest efficacy. Data from open-label and double-blind trials may be required to enhance our comprehension of the time frame for the remitted induction in those with moderate-severe active UC controlled with these agents.

Results from the baseline Mayo score, hs-CRP level, and patient weight subgroups demonstrated that patients with Mayo score $\geq 10$, elevated hs-CRP level ( $\geq 10 \mathrm{mg} / \mathrm{l}$ or $95.238 \mathrm{nmol} / \mathrm{l})$, and higher weight $(\geq 70 \mathrm{~kg})$ may have inflammatory subsequent that is not enough to give a worthy clinical remission by the dose of adalimumab utilized as a part of this trial. In this way, our information proposes the likelihood that a considerable extent of those with UC may require a larger dose of adalimumab to induce improvement, contrasted to Crohn's patients, however the reason for this remaining part still vague. Notwithstanding, patients with ulcerative colitis may require an expanded time of adalimumab course than those with Crohn's disorder to accomplish remission.

Regarding the significant and dangerous side effects of corticosteroids over a long-term administration, steroid-saving is a critical goal for persistent ulcerative colitis. In the ACT1 report, 
$61 \%$ of patients were on steroids during baseline; $21 \%$ of those who treated with infliximab were in steroid-free remission at 54 weeks (Rutgeerts et al., 2005). In the ULTRA2 trial, adalimumab treatment was related with significant rates of steroid stopping in patients taking steroids during baseline, with around $48 \%$ of patients getting to discontinued steroid at week 52 (Sandborn et al., 2012). In our study, no patient achieved remission with the steroid-free regimen for both groups throughout the time of induction and we did not evaluate the effects of adalimumab and infliximab during a maintenance long-term phase.

Previous information exhibited a comparable effect of adalimumab in European and Japan's patients with ulcerative colitis and Crohn's disease (Watanabe et al., 2012). Nonetheless, in one research accomplished in Japan, patients on adalimumab taking steroids during baseline may not virtually steroid-resistant. This fact was not observed when the study was finished, which is additionally agree with the known absence of long-term effect observed with steroid administration (Faubion et al., 2001).

In the current study, a little percent of adalimumab and infliximab patients were on immunosuppressive treatment. Whether the employment of immunosuppressants may contribute to the response rates for adalimumab or infliximab stays to be resolved. Further investigations are expected to decide the correct way by which immunosuppressive drugs may influence antiTNF antibodies, cytokine levels, and maintained improvement. Even though ACT and ACCENT cohort studies have indicated immunomodulator use to be identified with less immunogenicity and allergic reactions, yet not to enhance response, as contrasted to anti-TNF monotherapy (Lichtenstein et al., 2009).

Essential long-term results, including enhanced personal satisfaction, steroid withdrawal with the remission accomplishment, decrease in the incidence of hospitalization and colectomy happened in a higher rate with adalimumab maintenance regimen. The choice to progress with long-term treatment in patients with ulcerative colitis is, for the most part, depend on response to the course of induction (Dignass et al., 2012). The more prominent effect with time, combined with less incidence of general undesirable occasions in week 8 responders in respect to the patients getting biologic treatment, both help the ideal advantage/hazard ratio of maintenance regimen in patients showing a fast response to induction regimen.

In what capacity should the consequences of these data be applied to clinical practice? Adalimumab, given subcutaneously as self-administration, has not been examined on inpatients with serious ulcerative colitis who are not responded to IV steroids. It may offer an extra treatment choice for outpatients who did not endure infliximab or its infusion route that requires a special technique. In light of the subcutaneous injection method, adalimumab can be managed by patients at home, in this manner, staying away from more payment attributed to a higher frequency of hospital admission because of an intravenous mixture of infliximab.

The general safety issue of adalimumab and infliximab reported in the present trial was like that seen in different trials for these medications in patients with IBD (Colombel et al., 2009; Reinisch et al., 2011). Notably, comparable incidence rates were observed for severe, serious, and serious infectious adverse events in patients receiving adalimumab, compared with infliximab. The incidence of injection site reaction (mainly pain) and hematologic events (mainly leukopenia) was significantly higher in the infliximab group and may attribute to the IV route of administration for this agent. These events were mild and not required discontinuation of any agent.

One patient who got adalimumab and created tuberculosis in this research had other hazard factors for this disease; including expanded age (Rahier et al., 2009) and attendant high-amount corticosteroid utilize (Jick et al., 2006). The TB patient had experienced standard screening, skin test and radiologic assessment, reliable with the treatment algorithm. Tuberculosis has been seen in those on adalimumab with negative tests (Humira Package Insert, 2013) and may display newbeginning contaminations or false negative testing, which might be more probable in those on immunomodulators or corticosteroids (Rahier et al., 2009). Clinicians starting adalimumab in patients on immunosuppressive treatment or those with other hazard factors for contaminations ought to follow these patients for clinical findings, and should doubt of disease in those with manifestations of tuberculosis (high temperature, cough, weight reduction, etc).

Biotherapies are additionally connected with an expanded danger of malignancies, particularly lymphoma (Mason and Siegel, 2013). This is likely because blocking the function of TNF- alpha (tumor suppressive agent) and the apoptotic process. In the present trial, no patient endured lymphoma inside each arm.

Limitations of the current study were its relatively small sample sizes and short-term course (just the induction phase). Since ulcerative colitis is uncommon in Iraq compared to the Western nations, it has been hard to select a large sample of patients. Also, the design of this study was restricted by extensive inclusion/exclusion criteria, and some patients were withdrawn from the study by different reasons. Moreover, this research did not assess the adequacy of adalimumab and infliximab in patients who had beforehand gotten other biologic treatments.

Huge numbers of the biotherapies are immunogenic and patients as often as possible create antibodies against these medications, which can change their activity and tolerability. This issue is more successive with chimeric monoclonal antibodies, similar to infliximab, which is viewed as more immunogenic than completely humanized antibodies, as adalimumab (Hoentjen and Van, 2009). Even though in one report of adalimumab, the response of patients with ulcerative colitis to immunosuppressants and lack of antibody against adalimumab-platelet complex suggests that this biological agent can induce destruction of platelets by the formation of anti-platelet antibodies (immune-mediated thrombocytopenia) (Lee et al., 2015; Patil et al., 2012). So, future long-term, largescale studies may be required to measure antibodies against adalimumab or infliximab and their trough serum concentrations for the remitter and non-remitter patients, and for patients on concomitant immunosuppressive agents and those receiving just the biologic therapy. Also, and because of family history, genetic polymorphism, and variant human leukocyte antigens, studying the response rates of ulcerative colitis to the biologic therapy in different geographical regions may be required.

\section{CONCLUSION}

In conclusion, this real-life clinical trial demonstrated that adalimumab $(160 / 80 \mathrm{mg})$ and infliximab $(5 \mathrm{mg} / \mathrm{kg})$ were 
comparable in their effectiveness for inducing clinical remission and response in patients with moderate-severe ulcerative colitis who inadequately respond or intolerant to traditional management with oral corticosteroids and/or immunosuppressants. Both of the biologic agents were well tolerated, with an approach safety profile.

\section{ACKNOWLEDGMENTS}

The author would like to thank the Mustansiryiah University (www. uomustansiryiah.edu.iq), Baghdad-Iraq for its support in the present work. Special thanks from Dr. Bahir (dr.bahirrazzq@uomustansiriyah.edu.iq) also introduced to the gastroenterologists at Baghdad centers for diagnosis and followup, and the pharmacists Kanar Ahmad and Lubna Waleed for data collection.

\section{CONFLICT OF INTEREST}

The author declares that there has no conflict of interest.

\section{REFERENCES}

Afif W, Leighton JA, Hanauer SB. An open-label study of Adalimumab in patients with ulcerative colitis including those with prior loss of response or intolerance to infliximab. Inflamm Bowel Dis, 2009; $15: 1302-7$

Braegger CP, Nicholls S, Murch SH. Tumour necrosis factor alpha in stool as a marker of intestinal inflammation. Lancet, 1992; 339:89-91.

Chowers Y, Allez M. Efficacy of anti-TNF in Crohn's disease: how does it work? Curr Drug Targets, 2010; 11:138-42.

Cohen RD, Yu AP, Wu EQ. Systematic review: costs of ulcerative colitis in western countries. Aliment Pharmacol Ther, 2010; 31:693-07.

Colombel JF, Rutgeerts P, Reinisch W. Early mucosal healing with infliximab is associated with improved long-term clinical outcomes in ulcerative colitis. Gastroenterology, 2011; 141:1194-201.

Colombel JF, Sandborn WJ, Panaccione R. Adalimumab safety in global clinical trials of patients with Crohn's disease. Inflamm Bowel Dis, 2009; 15:1308-19.

Colombel JF, Sandborn WJ, Rutgeerts P. Adalimumab for maintenance of clinical response and remission in patients with Crohn's disease: the CHARM trial. Gastroenterology, 2007; 132:52-65.

Dignass A, Lindsay JO, Sturm A. Second European evidencebased consensus on the diagnosis and management of ulcerative colitis part 2: current management. J Croh Coli, 2012; 6:991-1030.

Faubion WA Jr, Loftus EV Jr, Harmsen WS. The natural history of corticosteroid therapy for inflammatory bowel disease: a populationbased study. Gastroenterology, 2001; 121:255-60.

Feagan BG, Sandborn WJ, Lazar A. Adalimumab therapy is associated with reduced risk of hospitalization in patients with ulcerative colitis. Gastroenterology, 2014; 146:110-2.

Ferrante M, Vermeire S, Fidder H. Long-term outcome after infliximab for refractory ulcerative colitis. J Croh Coli, 2008; 2:219-25.

Finniss DG, Kaptchuk TJ, Miller F. Biological, clinical, and ethical advances of placebo effects. Lancet, 2010; 375:686-95.

Frøslie KF, Jahnsen J, Moum BA. Mucosal healing in inflammatory bowel disease: results from a Norwegian population-based cohort. Gastroenterology, 2007; 133:412-22.

Hanauer SB, Sandborn WJ, Rutgeerts P. Human anti-tumor necrosis factor monoclonal antibody (adalimumab) in Crohn's disease: the CLASSIC-I trial. Gastroenterology, 2006;130:323-33.

Hinojosa J, Gomollo'n F, Garc1'a S. Efficacy and safety of shortterm Adalimumab treatment in patients with active Crohn's disease who lost response or showed intolerance to infliximab: a prospective, openlabel, multicentre trial. Aliment Pharmacol Ther, 2007; 25:409-18.
Hoentjen F, Van Bodegraven AA. Safety of anti-tumor necrosis factor therapy in inflammatory bowel disease. World J Gastroenterol, 2009; 15:2067-73.

Hudis N, Rajca B, Polyak S. The outcome of active ulcerative colitis treated with adalimumab. Gastroenterology, 2009; 136:683-5.

Humira [Japanese package insert]. AbbVie GK and Eisai Co, Ltd, Tokyo, Japan, 2013.

Irvine EJ, Feagan B, Rochon J. Quality of life: a valid and reliable measure of therapeutic efficacy in the treatment of inflammatory bowel disease. Canadian Crohn's Relapse Prevention Trial Study Group. Gastroenterology, 1994; 106:287-96.

Jick SS, Lieberman ES, Rahman MU. Glucocorticoid use, other associated factors, and the risk of tuberculosis. Arthr Rheum 2006; 55:19-26

Jürgens $\mathrm{M}$, Brand S, Laubender R. Disease activity and pANCA status determine early response to infliximab in ulcerative colitis. Gastroenterology, 2009; 136:680-5.

Langholz E. Current trends in inflammatory bowel disease: the natural history. Therap Adv Gastroenterol, 2010; 3:77-86.

Lee S, Chary M, Salehi I, Bansal R. Immune-mediated adalimumab-induced thrombocytopenia for the treatment of ulcerative colitis. Int J Pharm Pharm Sci, 2015; 7:456-8.

Lichtenstein GR, Diamond RH, Wagner CL. Clinical trial: benefits and risks of immunomodulators and maintenance infliximab for IBD-subgroup analyses across four randomized trials. Aliment Pharmacol Ther, 2009; 30:210-26.

Lichtenstein GR, Rutgeerts P. Importance of mucosal healing in ulcerative colitis. Inflamm Bowel Dis, 2010; 16:338-46.

Mason M, Siegel CA. Do inflammatory bowel disease therapies cause cancer? Inflamm Bowel Dis, 2013; 19:1306-21.

Murch SH, Braegger CP, Walker-Smith JA. Location of tumor necrosis factor alpha by immunohistochemistry in chronic inflammatory bowel disease. Gut, 1993; 34:1705-9.

Murch SH, Lamkin VA, Savage MO. Serum concentrations of tumor necrosis factor alpha in childhood chronic inflammatory bowel disease. Gut, 1991; 32:913-7.

Oussalah A, Laclotte C, Chevaux JB. Long-term outcome of adalimumab therapy for ulcerative colitis with intolerance or lost response to infliximab: a single-center experience. Aliment Pharmacol Ther, 2008; 28:966-72.

Patil US, Jaydeokar AV, Bandawane DD. Immunomodulators: a pharmacological review. Int J Pharm Pharm Sci, 2012; 4:30-6.

Peyrin-Biroulet L, Laclotte C, Roblin X. Adalimumab induction therapy for ulcerative colitis with intolerance or response to infliximab: an open-label study. World J Gastroenterol, 2007; 13:2328-32.

Rahier JF, Ben-Horin S, Chowers Y. European evidence-based Consensus on the prevention, diagnosis, and management of opportunistic infections in inflammatory bowel disease. J Croh Coli, 2009; 3:47-91.

Reinisch W, Sandborn WJ, Hommes DW. Adalimumab for induction of clinical remission in moderately to severely active ulcerative colitis: results of a randomized controlled trial. Gut, 2011; 60:780-7.

Rubin DT, Dubinsky MC, Panaccione R. The impact of ulcerative colitis on patients' lives compared to other chronic diseases: a patient survey. Dig Dis Sci, 2010; 55:1044-52.

Rutgeerts P, Sandborn WJ, Feagan BG. Infliximab for induction and maintenance therapy for ulcerative colitis. N Engl J Med, 2005; 353:2462-76.

Rutgeerts P, Vermeire S, Van Assche G. Mucosal healing in inflammatory bowel disease: impossible ideal or therapeutic target? Gut, 2007; 56:453-5.

Sandborn WJ, Rutgeerts P, Enns R. Adalimumab induction therapy for Crohn disease previously treated with infliximab: a randomized trial. Ann Intern Med, 2007; 146:829-38.

Sandborn WJ, Rutgeerts P, Feagan BG. Colectomy rate comparison after treatment of ulcerative colitis with placebo or infliximab. Gastroenterology, 2009; 137:1250-60. 
Sandborn WJ, van Assche G, Reinisch W. Adalimumab induces and maintains clinical remission in patients with moderate-to-severe ulcerative colitis. Gastroenterology, 2012; 142:257-65.

Sandborn WJ, Van Assche GA, Thakkar R. Adalimumab improves health-related quality of life for 52 weeks in patients with ulcerative colitis. Gastroenterology, 2011; 140:263-5.

Sartor RB. Cytokines in intestinal inflammation: pathophysiological and clinical considerations. Gastroenterology, 1994;106:533-9.

Schroeder KW, Tremaine WJ, Ilstrup DM. Coated oral 5 -aminosalicylic acid therapy for mildly to moderately active ulcerative colitis. A randomized study. N Engl J Med, 1987; 317:1625-9.

Senent S, Martín-Arranz M, Merino L. Infliximab in ulcerative colitis: concomitant cytomegalovirus infection, lifes quality, PPD positive have an influence on the treatment with infliximab. Gastroenterology, 2009; 136:682-6.

Targan SR, Hanauer SB, van Deventer SJ. A short-term study of chimeric monoclonal antibody cA2 to tumor necrosis factor- $\alpha$ for Crohn's disease. N Engl J Med, 1997; 337:1029-35.
Teshima CW, Thompson A, Dhanoa L. Long-term response rates to infliximab therapy for Crohn's disease in an outpatient cohort. Can J Gastroenterol, 2009; 23:348-52.

Watanabe M, Hibi T, Lomax KG. Adalimumab for the induction and maintenance of clinical remission in Japanese patients with Crohn's disease. J Croh Coli, 2012; 6:160-73.

William J. Sandborn, Atsushi Sakuraba, Anthony Wang. Comparison of real-world outcomes of adalimumab and infliximab for patients with ulcerative colitis in the United States. Curr Med Res Opin, 2016; 32:1233-41.

How to cite this article:

Mshimesh BAR. Studying activity and toxicity of different TNF-alpha inhibitors in controlling patients with active ulcerative colitis. J Appl Pharm Sci, 2019; 9(02):087-096. 